http://revistas.ucr.ac.cr/index.php/ingenieria

www.ucr.ac.cr / ISSN 1409-2441
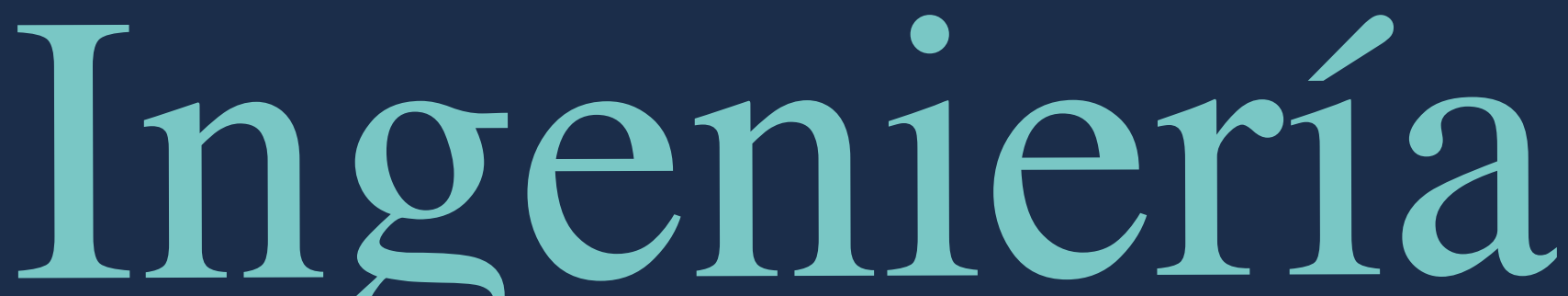

Revista de la Universidad de Costa Rica JULIO / DICIEMBRE 2019 - VOLUMEN 29 (2)

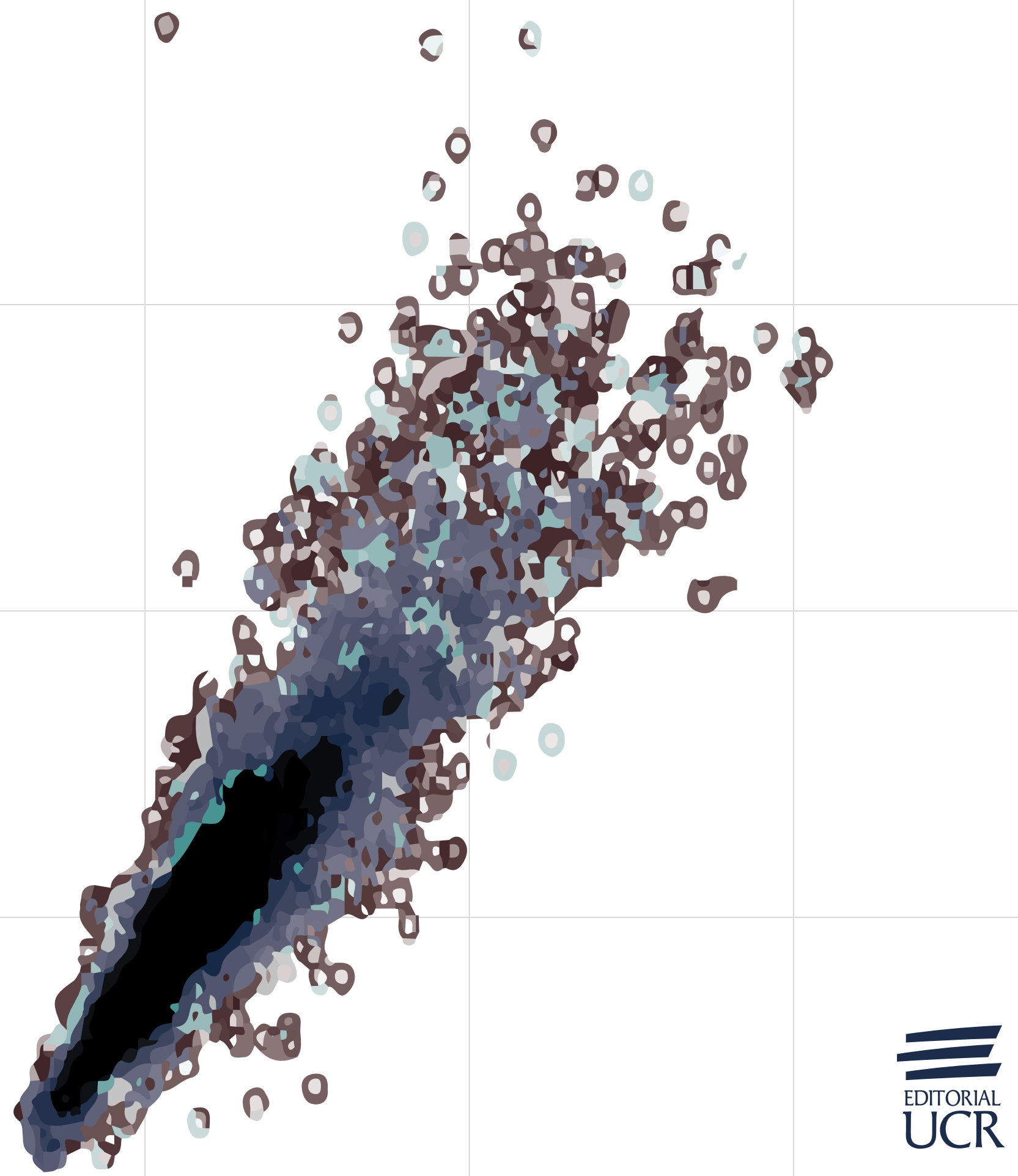




\title{
Implementación de tablas de corrección al volumen del tanque cilíndrico horizontal inclinado, compatibles con la norma ISO 12917:2017
}

\author{
Implementation of volume correction tables of the inclined horizontal \\ cylindrical tank, compatible with ISO 12917: 2017
}

José Rafael Mora Casal

Consultor privado, Costa Rica

Correo:joserafaelmora@gmail.com

René Mora Casal

Escuela de Química, Universidad Nacional (UNA), Costa Rica

Correo:rene.mora.casal@una.cr

Recibido: 9 de marzo $2019 \quad$ Aceptado: 29 de julio 2019

\begin{abstract}
Resumen
Se presenta un método analítico, matemáticamente exacto y explícito para implementar la corrección por inclinación que se describe en la norma ISO 12917:2017 para calibración de tanques horizontales cilíndricos. El método es superior al método gráfico propuesto en la norma hasta 2017 y mejora lo propuesto en la norma actual. Se discuten y aclaran varias de las dificultades que presenta el método de corrección de la norma. Como aporte novedoso, se produjeron dos tablas numéricas con valores de corrección por inclinación, equivalentes a la Figura A-1 de la norma ISO 12917:2002 y las cuales no se han reportado previamente.
\end{abstract}

\section{Palabras clave:}

Calibración de tanques, transferencia en custodia, tanque horizontal inclinado, corrección por inclinación, norma ISO 12917.

\begin{abstract}
An analytical, mathematically accurate and explicit method to implement tilt correction, as described in the ISO 12917:2017 standard for calibration of horizontal cylindrical tanks, is presented. The method is superior to the graphical method proposed in the standard up to 2017 and improves the one proposed in the current standard. Several of the difficulties presented by the correction method of the standard are discussed and clarified. As a new contribution, two numerical tables were produced with tilt correction values, equivalent to Figure A-1 of ISO 12917:2002 and which have not been previously reported.
\end{abstract}

Keywords:

Tank calibration, custody transfer, tilted horizontal tank, tilt correction, ISO 12917 standard. 


\section{INTRODUCCIÓN}

En la industria química y alimentaria es común el uso de tanques horizontales para el almacenamiento de productos químicos. Durante las operaciones de inventario y transferencia en custodia de petróleo y otros fluidos de interés comercial, la cantidad de líquido en los tanques debe ser conocida con exactitud y precisión, ya que existen implicaciones comerciales y legales por cualquier error que se presente durante los procesos de compra y venta de los productos almacenados. Las implicaciones prácticas de esto son importantes: en el caso de la Refinadora Costarricense de Petróleo, por ejemplo, una diferencia de $0,1 \%$ durante el bombeo de gasolina a 2271 litros por minuto, a un precio neto de 342,56 colones por litro, representaría al año 409 millones de colones o USD 710000.

Lo anterior determina que todos los tanques de almacenamiento requieran una tabla de incrementos de volumen contra la altura del nivel de líquido, conocidas como tablas de calibración. Para efectos legales y comerciales, dicha tabla debe ser calculada usando el procedimiento estándar de calibración internacionalmente reconocido, descrito en la norma ISO 12917. Dicha norma explica la forma de calibrar un tanque cilíndrico horizontal y cómo calcular la tabla de incrementos de volúmenes; esto es necesario porque el tanque, en realidad, no es un cilindro perfecto, y la norma indica lo que procede para calcular un volumen confiable, incluyendo varias correcciones como son la inclinación (tilt), la temperatura y la presión de operación.

El presente estudio se enfocará en la corrección por inclinación, presentando una alternativa al método gráfico, ya que la norma es muy escueta acerca de cómo aplicarla. Se propondrá un método para el cálculo del volumen de un tanque horizontal inclinado que resuelva las limitaciones y problemas asociados con el método de cálculo gráfico establecido y que mantenga la compatibilidad con la norma vigente. Asimismo, se generarán tablas que permitan leer la corrección por inclinación (tilt) con mayor exactitud que el método gráfico.

Dentro del alcance de este estudio no se contempla el cálculo de la incertidumbre en el cálculo del volumen por dos razones: (i) el método propuesto es matemáticamente exacto y (ii) la norma actual incluye un apéndice (el Apéndice C) dedicado a presentar las ecuaciones para el cálculo de la incertidumbre del volumen y que se resumen en la ecuación (C.4) de la norma:

$$
u_{V}^{2}=\left(u_{R} \frac{\delta V}{\delta R}\right)^{2}+\left(u_{W} \frac{\delta V}{\delta W}\right)^{2}+\left(u_{W_{g}} \frac{\delta V}{\delta W_{g}}\right)^{2}+\left(u_{\gamma} \frac{\delta V}{\delta \gamma}\right)^{2}
$$

La ecuación anterior implica que la incertidumbre del volumen depende de las incertidumbres del radio del tanque $(\mathrm{R})$, del ángulo de inclinación $(\gamma)$, de la longitud del tanque $(\mathrm{W})$ y de la longitud entre el punto más bajo del cuerpo del tanque y la posición de medición (gauge position) (Wg). Cabe mencionar que dicha ecuación es ilustrativa y por eso no será numerada. 


\section{ANTECEDENTES DE LA NORMA ISO 12917:2017 Y LA CORRECCIÓN POR INCLINACIÓN}

La norma vigente para calibración de tanques horizontales es la ISO 12917:2017 Petroleum and liquid petroleum products -- Calibration of horizontal cylindrical tanks -- Part 1: Manual methods, la cual se basa en la norma API 2551 Standard Method for Measurement and Calibration of Horizontal Tanks del año 1965, donde se recomendó por primera vez la corrección por inclinación. La norma API 2551 fue ratificada por muchos años, y luego se convirtió en la norma ISO 12917:2002, cuya revisión más reciente es del año 2017. Tanto la norma API como la norma ISO, hasta el año 2017, indicaban que la corrección por inclinación (tilt) se debía obtener de un gráfico, basado en el método propuesto por W. L. Coats en 1948, quien resolvió por primera vez el problema del volumen del tanque horizontal inclinado (ISO, 2002; API, 1965; Coats, 1948). El gráfico original era la Figura 8 de la norma API 2551, que corresponde a la Figura A.1 de la norma del 2002; un gráfico equivalente -elaborado con los métodos del presente estudio, ya que el original posee derechos de autor- se puede observar en la Figura 1. El porcentaje de corrección se calcula así:

$$
\% \text { corrección }=100 \times\left(V_{H T}-V_{H}\right) / V_{F}
$$

donde $V_{H T}, V_{H} y V_{F}$ representan los volúmenes del tanque horizontal, tanque horizontal inclinado (tilted) y tanque lleno, respectivamente. $\mathrm{V}_{\mathrm{HT}}$ representa el valor verdadero y correcto del volumen de líquido.

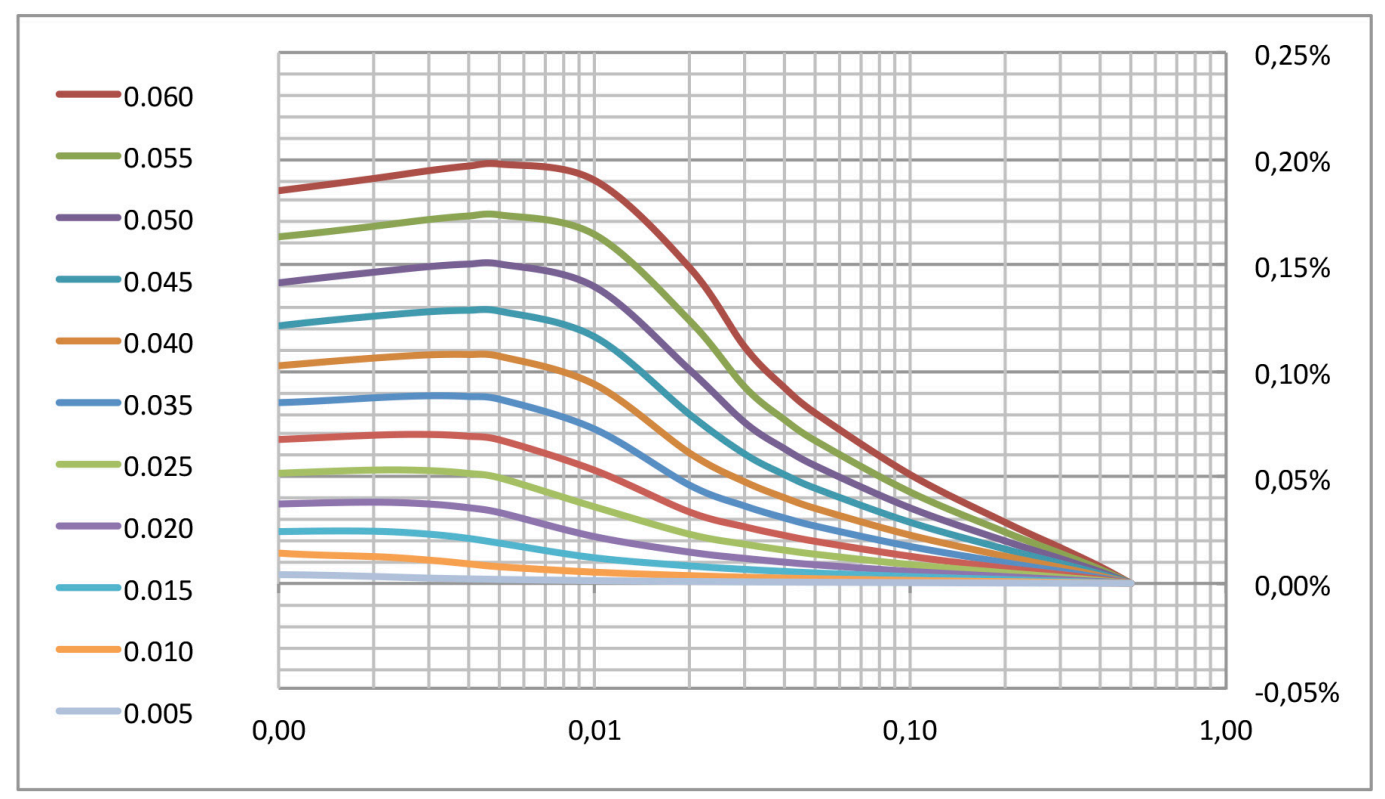

Figura 1. Gráfico para calcular la corrección por inclinación.

Fuente: elaboración propia. 
La Figura A-1 en la norma ISO 12917:2002 presenta dos serios problemas: (i) la corrección debe interpolarse del gráfico en cuestión, en una escala logarítmica, esto tiene como consecuencia que el valor leído es aproximado y su incertidumbre es desconocida; (ii) no es práctica para usarla en programas de cálculo de tablas de calibración, porque no se da la fórmula usada para estimar las correcciones. Implica usar tablas aproximadas e ingresar valores manualmente. Estos problemas se agravan si consideramos que muchos de los usuarios de la norma ISO 12917 no son ingenieros -de hecho, no es un requisito para usar la norma-. Esto crea una serie de limitantes a la hora de aplicar la corrección al volumen del tanque, tales como:

a) El usuario crea ecuaciones empíricas para la corrección, aproximadas y no exactas.

b) Se usan métodos manuales o artesanales para incluir las correcciones en los programas de cálculo de tablas volumétricas, lo cual es muy ineficiente.

c) Algunos usuarios deciden no usar corrección del todo, lo cual impacta la confiabilidad de las tablas generadas y puede tener consecuencias legales y comerciales.

Los problemas anteriores se resuelven si se propone o desarrolla un método que sea analítico y matemáticamente exacto; por tanto, superior al método gráfico. La versión más reciente de la norma, ISO 12917:2017, reemplaza el gráfico por un método analítico equivalente al que se propone en este estudio; sin embargo, no se resuelve el problema porque el método propuesto no es suficientemente claro e incluso posee algunos errores, p. ej. de tipo dimensional en las ecuaciones (6), (7) y (11) de la Sección 10.2.3.2.2., lo cual impide usarlas y determina que los usuarios sigan dependiendo del método gráfico. Específicamente, las ecuaciones (6) y (7) de la norma tienen la forma siguiente:

$$
h_{t}=h_{b e}+\left(1 / \cos (\gamma)+\delta H_{g}\right)-W \cdot \tan (\gamma)=\mathrm{h}_{\mathrm{b}}-W \tan (\gamma)
$$

El término $1 / \cos (\gamma)$ es dimensionalmente inconsistente, el término correcto sería $\mathrm{H}^{\prime} / \cos (\gamma)$ usando la notación de la norma. Por otra parte, la ecuación (11) de la norma tiene la forma siguiente:

$$
V=\frac{R^{3}}{\tan (\gamma)}\left(q_{b}-q_{t}\right)+\pi\left(\frac{D_{i}}{2}\right)^{2} \cdot W_{c}
$$

En el segundo término de esta ecuación debe ir Wnft en lugar de Wc para que sea correcta. Estas ecuaciones son ilustrativas y por lo anterior no serán numeradas.

\section{APORTES Y ALCANCE DEL ESTUDIO}

El problema de calcular el volumen de un tanque horizontal inclinado ha sido resuelto por diferentes autores (por ejemplo, Khaisongkram y Banjerdpongchai, 2004; Xie et al. 2012; Alonso-Preciado et al., 2008; Li et al., 2013). Las soluciones anteriores se enfocan principalmente en 
los aspectos matemáticos del problema, y no hacen referencia a la norma de calibración. La fórmula obtenida para el volumen del tanque se indefine para ciertos valores de la altura, lo cual obliga a considerar varios casos. Los tanques horizontales pueden ser rectangulares, cilíndricos o elipsoidales; con tapas planas, semiesféricas o torisféricas, lo cual multiplica el número de casos a considerar. Para el presente estudio se considerará el tanque como un cilindro horizontal de tapas planas, esto es conforme con la norma y con el autor del método (Coats, 1948). Las razones de lo anterior son tres: (i) la norma cubre solamente los tanques cilíndricos; (ii) los tanques cilíndricos representan la mayoría de los tanques encontrados en el mercado y (iii) la misma norma indica que los volúmenes de las tapas curvas no se corrigen -solo se calculan y se suman como si no hubiera inclinación- porque el error se considera despreciable (Coats, 1948; ISO, 2017, Sección 10.2.3.3.).

Asimismo, se considerará una inclinación, medida como el cociente E/D, no mayor que 6\% por razones que se explicarán más adelante. La norma ISO 12917:2002 asume que la medición se realiza en el punto medio del tanque y no explica cómo se procede cuando el punto de medición no está en dicho punto, esto también se debe aclarar.

Este trabajo espera aportar varias soluciones a los problemas mencionados:

1) Presentar una fórmula explícita, compatible con la nomenclatura de la norma ISO 12917:2017 vigente, para generar los incrementos volumétricos con mayor exactitud que el método gráfico.

2) Aclarar el método que se debe aplicar a los casos en los que el punto de medición es distinto al punto medio del tanque (L/2).

3) Aclarar los métodos aplicables en los casos para los que la fórmula del caso típico se indefine.

4) Desarrollar tablas de correcciones por inclinación, equivalentes a la Figura A-1 de la norma ISO 12917:2002, que no existen actualmente y que serían más exactas que el gráfico.

\section{METODOLOGÍA}

En la Figura 2, se presenta el tanque horizontal inclinado con las variables de estudio.

El tanque tiene longitud $\mathrm{L}$ y diámetro $\mathrm{D}=2 \mathrm{R}$. Las alturas de líquido en los extremos el tanque, medidas según el eje vertical del tanque, son $\mathrm{h}_{1} \mathrm{y} \mathrm{h}_{2}$. El punto de medición se ubica a una distancia u del extremo más alto, según el eje horizontal del tanque. La altura que se mediría en el punto de medición, si el tanque fuera perfectamente horizontal, sería $\mathrm{H}$, mientras que la altura que realmente se mide es H'. La inclinación del tanque determina que la diferencia de alturas entre los extremos es E, mientras que la proyección de la longitud del tanque sobre la horizontal es L'. La relación entre H y H' no es simple; sin embargo, en una aproximación de primer orden elaborada por los autores se puede escribir:

$$
H=H^{\prime} \times \frac{L^{\prime}}{L}=H^{\prime} \times \sqrt{\left(\frac{L^{2}-E^{2}}{L^{2}}\right)}=H^{\prime} \times \sqrt{1-\left(\frac{E}{L}\right)^{2}}
$$




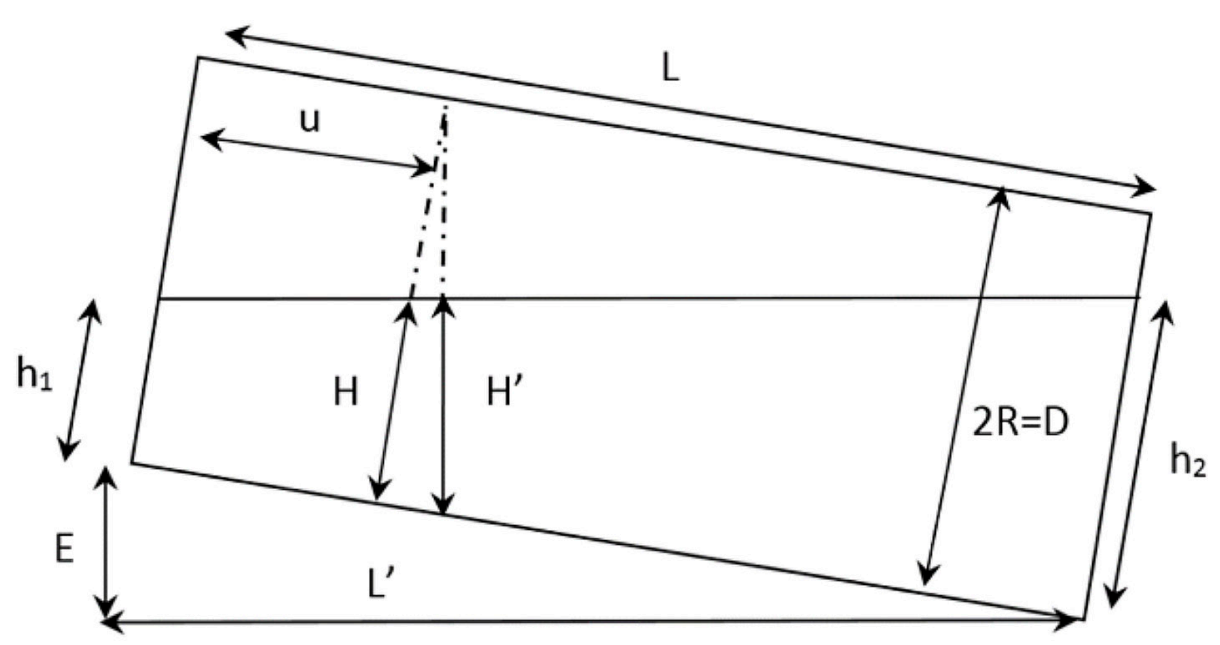

Figura 2. Tanque horizontal inclinado. Fuente: elaboración propia.

Se puede demostrar que para una inclinación E/L igual o menor a 6\%, el segundo factor de la ecuación (1) se puede considerar igual a la unidad con un error menor que $0,18 \%$. Por esta razón, a partir de este punto se considerará $\mathrm{H}=\mathrm{H}^{\prime}$ para efectos de este estudio, esto equivale a definir $\mathrm{L}=\mathrm{L}$ '. El análisis se puede plantear ahora en términos de la Figura 3, donde el tanque se ha "enderezado".

ç

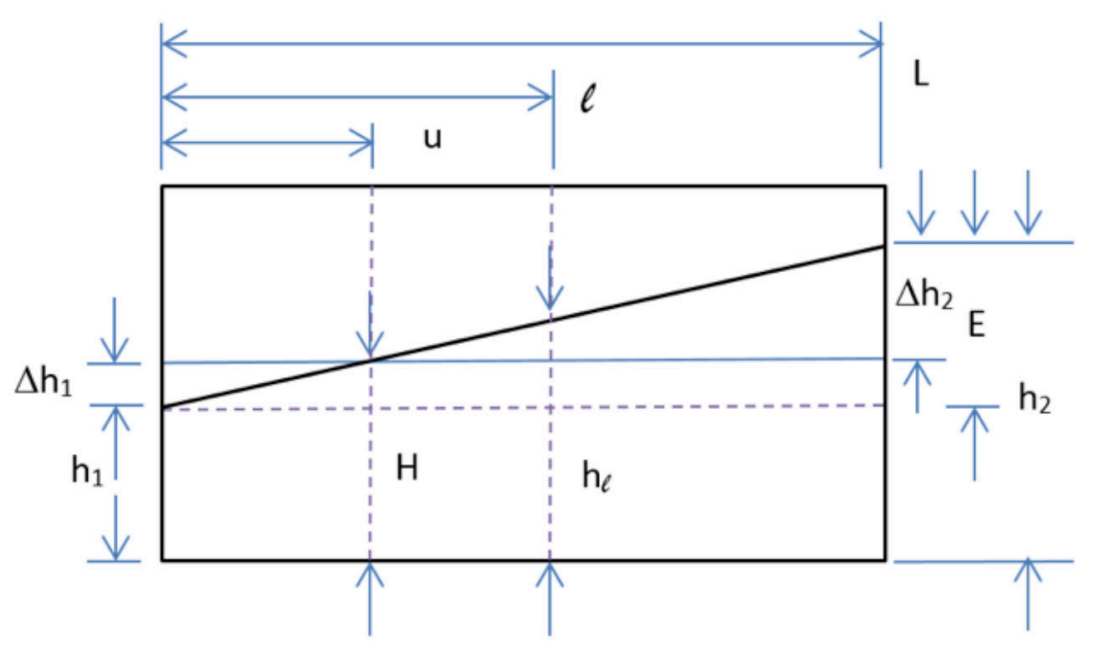

Figura 3. Relación entre alturas y posición en el tanque inclinado. Fuente: elaboración propia.

La primera observación de la figura anterior es que, si el punto de medición se ubica a medio tanque (es decir, $\mathrm{u}=\mathrm{L} / 2)$ y además el tanque está medio lleno $(\mathrm{h} / \mathrm{D}=0,50)$, entonces las diferencias de volumen a ambos lados del tanque, representados por los triángulos a cada lado del eje 1 $=\mathrm{u}$, serían iguales y no se requiere corrección. Esto se representa en la Figura 1 por el hecho de que la corrección es cero cuando $h / D=0,50$. Para cualquier otro valor de $h / D$, las diferencias de 
volumen no serán iguales, esto determina que la corrección será positiva si h/D $<0,50$ y negativa si h/D >0,50. Otra observación es que, cuando el volumen de líquido en el tanque es mayor que el $50 \%$ del total, el cálculo del volumen de líquido se puede considerar complementario al caso correspondiente cuyo volumen es menor al 50\% del total. Estas observaciones también se reflejan en la forma en que está construida la Figura 1, así como en su uso según la norma, constituyendo la base del método que se va a proponer. Con base en la geometría de la Figura 3 se cumple lo siguiente:

$$
\begin{aligned}
& h_{l}=h_{l}+\Delta h_{l} \\
& h_{2}=h_{l}+E
\end{aligned}
$$

$$
\frac{\Delta h_{l}}{E}=\frac{l}{L}
$$

Estas ecuaciones permiten establecer las relaciones entre las diferentes alturas:

$$
\begin{aligned}
& h_{l}=h_{1}+l \cdot \frac{E}{L} \\
& h_{1}=H-u \cdot \frac{E}{L} \\
& h_{2}=H+(L-u) \cdot \frac{E}{L} \\
& V_{H}=A \times L \\
& V_{H}=L \times\left[R^{2} \times \arccos \left(\frac{R-H}{R}\right)-(R-H) \sqrt{2 R H-H^{2}}\right]
\end{aligned}
$$

El volumen de líquido del tanque horizontal inclinado se obtiene de la siguiente manera:

$$
\begin{gathered}
V_{H T}=\int_{0}^{L} A \times d l=\frac{L}{E} \int_{h_{1}}^{h_{2}} A \times d h \\
V_{H T}=\frac{L}{E} \int_{h_{1}}^{h_{2}}\left[R^{2} \times \arccos \left(\frac{R-h}{R}\right)-(R-h) \sqrt{2 R h-h^{2}}\right] d h
\end{gathered}
$$


La solución de la integral resulta en la siguiente fórmula:

$$
\begin{aligned}
& V_{H T}=\frac{L R^{3}}{E} \times\left[\sqrt{\frac{h}{R} \times\left(2-\frac{h}{R}\right)}-\left(1-\frac{h}{R}\right) \times \arccos \left(1-\frac{h}{R}\right)-\frac{1}{3} \times\left(\sqrt{\frac{h}{R} \times\left(2-\frac{h}{R}\right)}\right)^{3}\right]_{h_{1}}^{h_{2}} \\
& V_{F}=\pi R^{2} L
\end{aligned}
$$

El término entre corchetes de la ecuación (13) equivale a la ecuación (10) de la Sección 10.2.3.2.3. de la norma vigente. El término $\left(L R^{3} / E\right)$ equivale al término $R^{3} / \tan \gamma$ de la ecuación (11) de la misma sección de la norma. Las anteriores fórmulas permiten calcular el factor de corrección requerido.

\section{ADAPTACIÓN A LA NORMA Y ANÁLISIS DE CASOS}

El primer paso para adaptar las ecuaciones anteriores a la norma vigente es hacer una traslación de la altura medida en el punto $l=\mathrm{u}$ al punto $l=\mathrm{L} / 2$. Esto se consigue mediante la aplicación de las ecuaciones (4), (6) y (7), donde los valores de $h_{1} \mathrm{y} \mathrm{h}_{2}$ son los mismos calculados anteriormente:

$$
\begin{aligned}
& H_{0,5}=h_{1}+\frac{L}{2} \cdot \frac{E}{L}=h_{1}+\frac{E}{2}=h_{2}-\frac{E}{2} \\
& H_{0,5}=H-\left(\frac{L}{2}-u\right) \cdot \frac{E}{L}
\end{aligned}
$$

Hasta ahora se ha considerado que se conoce el valor de E. Dicho valor se puede medir directamente para un tanque sobre el suelo, o bien se requieren al menos dos puntos de medición para un tanque subterráneo y aplicar una proporción geométrica. Ahora corresponde definir tres casos para aplicar o adaptar la fórmula obtenida, ecuación (13), los cuales se ilustran en la Figura 4.
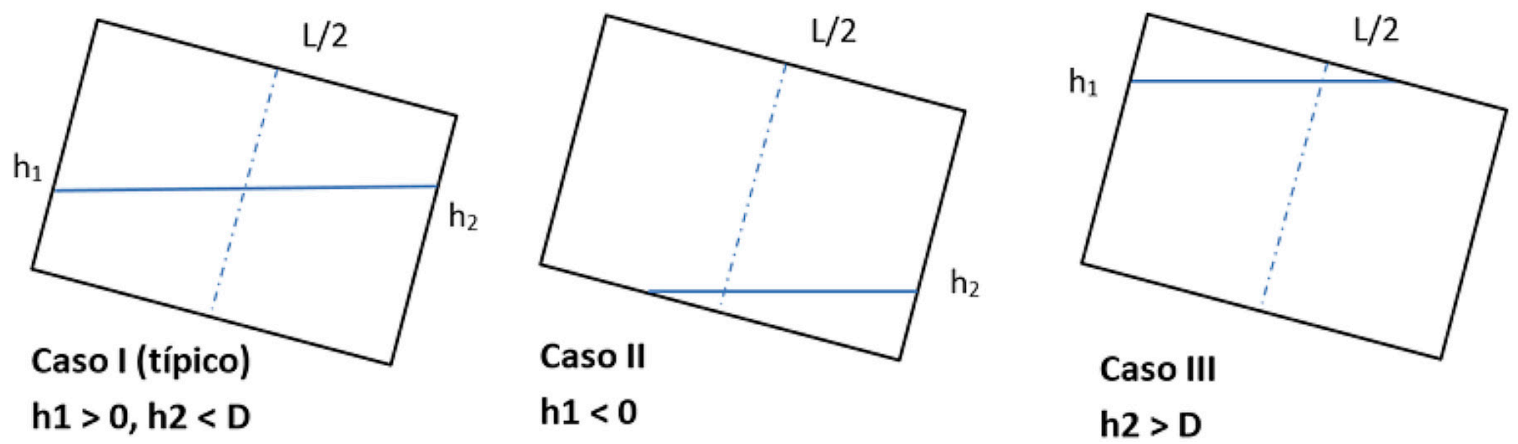

Figura 4. Posibles casos para el tanque horizontal inclinado. Fuente: elaboración propia. 
A continuación se propone cómo se calcularía el volumen de líquido del tanque horizontal inclinado $\left(\mathrm{V}_{\mathrm{HT}}\right)$ para cada uno de los tres casos:

Caso I: aplicación directa de la ecuación (13).

Caso II: aplicación directa de la ecuación (13) con límite inferior $\mathrm{h}_{1}=0$.

Caso III: aplicación directa de la ecuación (13) con límite superior $\mathrm{h}_{2}=2 \mathrm{R}=\mathrm{D}$. Además, se debe sumar el volumen de un cilindro, calculado de la siguiente forma:

$$
\frac{L}{E} \int_{2 R}^{h_{2}}\left(\pi R^{2}\right) d h=\left(\pi R^{2}\right)\left(\frac{L}{E}\right)\left(h_{2}-2 R\right)
$$

Una forma alternativa y equivalente de resolver el Caso III es verlo como el complemento del Caso I, esto es conforme con otros autores (Coats, 1948; Xie et al., 2012); bajo esta perspectiva, al volumen total $\left(\mathrm{V}_{\mathrm{F}}\right)$ se restaría el volumen del espacio vacío, calculado a partir de la ecuación (13) mediante el siguiente cambio de variable:

$$
\begin{array}{r}
h_{2}^{\prime}=2 R-h_{1} \\
V_{H T}=V_{F}-\frac{L}{E} \int_{0}^{h_{2}^{\prime}} A d h^{\prime}
\end{array}
$$

\section{ANÁLISIS Y DISCUSIÓN}

Con el fin de agilizar los cálculos, se recomienda adoptar una idea originalmente planteada por (Coats, 1948): consiste en definir una función Q(h) de modo que

$$
\begin{gathered}
Q(h)=\sqrt{\frac{h}{R} \times\left(2-\frac{h}{R}\right)}-\left(1-\frac{h}{R}\right) \times \arccos \left(1-\frac{h}{R}\right)-\frac{1}{3} \times\left(\sqrt{\frac{h}{R} \times\left(2-\frac{h}{R}\right)}\right)^{3} \\
\int_{h_{1}}^{h_{2}} A \times d h=Q\left(h_{2}\right)-Q\left(h_{1}\right)
\end{gathered}
$$

La ecuación (20) equivale a la ecuación (10) de la Sección 10.2.3.2.3. de la norma vigente. La ecuación (21) equivale al término $\left(\mathrm{q}_{\mathrm{b}}-\mathrm{q}_{\mathrm{t}}\right)$ en la ecuación (11) de la misma sección. La función 
$\mathrm{Q}(\mathrm{h})$ adopta un valor de cero para $\mathrm{h}=0$ y un valor de $\pi=3,14159265$ para $h=2 \mathrm{R}=\mathrm{D}$, como se ve en la Figura 5.

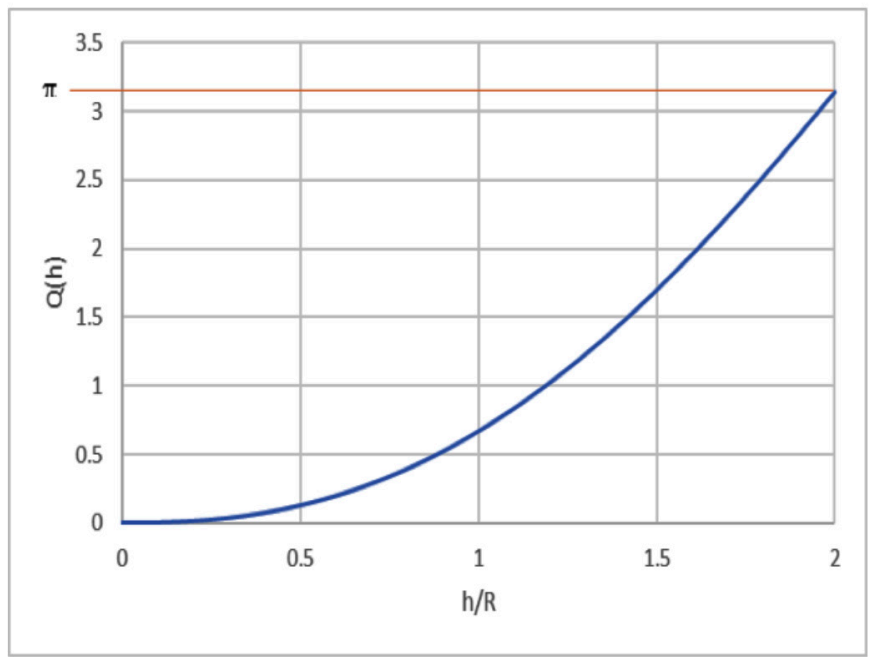

Figura 5. Función auxiliar Q(h). Fuente: elaboración propia.

Anteriormente se indicó que la norma ISO 12917:2002 adopta un valor máximo para el cociente E/D de 0,06. Las razones por las que se adopta E/D como parámetro tienen su origen en el autor del método de calibración de tanques (Coats, 1948), quien determinó que el volumen del tanque depende solamente de dos parámetros, h/D y E/D. Esto se refleja en la forma particular como este autor escribe la fórmula del volumen, combinando las ecuaciones (13), (14) y (21):

$$
V_{H T}=\frac{V_{F}}{2 \pi \cdot(E / D)} \times\left[Q\left(h_{2}\right)-Q\left(h_{1}\right)\right]
$$

Un parámetro que está más relacionado con el ángulo de inclinación del tanque es E/L, cociente que representa una razón trigonométrica $(\tan \gamma)$. Dado que $E / D=(E / L)(L / D)=(L / D)(\tan \gamma)$ y normalmente L será mayor que $\mathrm{D}$ para un tanque horizontal, entonces $\mathrm{E} / \mathrm{L}<<0,06$ en general. Esto valida las suposiciones $\mathrm{L}=\mathrm{L}$ ' y $\mathrm{H}=\mathrm{H}^{\prime}$ hechas anteriormente.

Para calcular el volumen desde el tanque completamente vacío hasta el tanque completamente lleno, conviene permitir que $\mathrm{H}$ adopte valores negativos y mayores que $\mathrm{D}$. Los resultados para valores selectos de $\mathrm{h}$ y $\mathrm{R}=1 \mathrm{~m}, \mathrm{~L}=6 \mathrm{~m}, \mathrm{E}=0,12 \mathrm{~m} \mathrm{y} \mathrm{u}=1,2 \mathrm{~m}$ se observan en el Tabla 1 y la Figura 6:

En la Tabla 1, se observa el orden en que conviene hacer los cálculos para la aplicación del método propuesto. Primero se presentan los valores de $\mathrm{H}_{0,5}, \mathrm{~h}_{1} \mathrm{y} \mathrm{h}_{2}$ según las ecuaciones (15) y (16); posteriormente se cambian los valores de $h_{1} \mathrm{o}$ de $\mathrm{h}_{2}$ según sean aplicables los Casos I, II y III. Se calcula la función $\mathrm{Q}(\mathrm{h})$ para los nuevos valores de $\mathrm{h}_{1} \mathrm{y}_{2} \mathrm{y}$ se restan, siguiendo las ecuaciones 
(20) y (21). Finalmente, se calculan la corrección para el Caso III, ecuación (17) y el volumen de líquido $\mathrm{V}_{\mathrm{HT}}$, ecuación (13). Se puede demostrar que último volumen de la tabla coincide con el valor calculado para el tanque lleno, ecuación (14), cuyo valor es $18,850 \mathrm{~m}^{3}$. No es necesario calcular volumen del tanque horizontal VH ni porcentaje de corrección porque se está calculando directamente el volumen del tanque inclinado.

Tabla 1. Volúmenes tanque horizontal inclinado, $\mathrm{E} / \mathrm{L}=0,02 \mathrm{y}$ u/L $=0,2$. Fuente: elaboración propia.

\begin{tabular}{|c|c|c|c|c|c|c|c|c|c|c|c|}
\hline & & & & & & & & & $\begin{array}{l}\text { Corr } \\
\text { Caso }\end{array}$ & VHT & \\
\hline $\mathrm{H}$ & $\mathrm{H}(0,5)$ & h1 & h2 & h1 & $\mathrm{Q}(\mathrm{h} 1)$ & h2 & $\mathrm{Q}(\mathrm{h} 2)$ & $\mathrm{Q}(\mathrm{h} 2)-\mathrm{Q}(\mathrm{h} 1)$ & III & $\left(\mathrm{m}^{3}\right)$ & Comentario \\
\hline$-0,096$ & $-0,060$ & $-0,120$ & 0,000 & 0,000 & 0,00000 & 0,000 & 0,00000 & 0,00000 & 0,00000 & 0,00000 & tq vacío \\
\hline$-0,036$ & 0,000 & $-0,060$ & 0,060 & 0,000 & 0,00000 & 0,060 & 0,00066 & 0,00066 & 0,00000 & 0,03304 & \\
\hline 0,000 & 0,036 & $-0,024$ & 0,096 & 0,000 & 0,00000 & 0,096 & 0,00213 & 0,00213 & 0,00000 & 0,10657 & $\begin{array}{l}\text { volumen } \\
\text { inaccesible }\end{array}$ \\
\hline 0,024 & 0,060 & 0,000 & 0,120 & 0,000 & 0,00000 & 0,120 & 0,00371 & 0,00371 & 0,00000 & 0,18568 & \\
\hline 0,964 & 1,000 & 0,940 & 1,060 & 0,940 & 0,57602 & 1,060 & 0,76451 & 0,18850 & 0,00000 & 9,42478 & $\begin{array}{l}\text { tanque medio } \\
\text { lleno }\end{array}$ \\
\hline 1,000 & 1,036 & 0,976 & 1,096 & 0,976 & 0,62954 & 1,096 & 0,82667 & 0,19713 & 0,00000 & 9,85643 & \\
\hline 1,904 & 1,940 & 1,880 & 2,000 & 1,880 & 2,76832 & 2,000 & 3,14159 & 0,37328 & 0,00000 & 18,6639 & \\
\hline 1,964 & 2,000 & 1,940 & 2,060 & 1,940 & 2,95376 & 2,000 & 3,14159 & 0,18783 & 9,42478 & 18,8165 & \\
\hline 2,000 & 2,036 & 1,976 & 2,096 & 1,976 & 3,06626 & 2,000 & 3,14159 & 0,07533 & 15,0796 & 18,8462 & \\
\hline 2,024 & 2,060 & 2,000 & 2,120 & 2,000 & 3,14159 & 2,000 & 3,14159 & 0,00000 & 18,8496 & 18,8496 & tanque lleno \\
\hline
\end{tabular}

Observando la Figura 1, se puede notar que, cuando $\mathrm{h}_{2}=0 \mathrm{~m}$, el tanque estaría completamente vacío, cuando $h_{1}=2 \mathrm{~m}$ el tanque estaría completamente lleno y cuando $\mathrm{H}_{0,5}=1 \mathrm{~m}$ el tanque está medio lleno, lo cual coincide con las primeras observaciones hechas en este documento. Observando la Figura 6, el volumen está representado por una curva simétrica con respecto a una línea horizontal igual al 50\% del volumen total del tanque; sin embargo, la línea de simetría vertical no coincide con la mitad del valor máximo de $\mathrm{H}$, no está “centrada". Cuando $\mathrm{H}=0$, la inclinación del tanque determina un pequeño volumen de líquido que es inaccesible, en el sentido que no se puede medir, pero el presente método permite estimarlo: en este caso su valor es de $0,1065 \mathrm{~m}^{3} \mathrm{o}$ de 106,5 litros. Asimismo, cuando $\mathrm{H}=\mathrm{D}$, queda un pequeño volumen de espacio vacío que también resulta inaccesible, porque de seguirse llenado el tanque el líquido se derramaría por la boca de medición; el volumen en este punto sería el volumen máximo o capacidad del tanque inclinado, en el ejemplo sería $18,846 \mathrm{~m}^{3}$. Este método permite también estimar dicho volumen vacío como $(18,850-18,846)=0,0034 \mathrm{~m}^{3}$ o 3,4 litros. Estos volúmenes están representados por los círculos rojos en la Figura 6. 


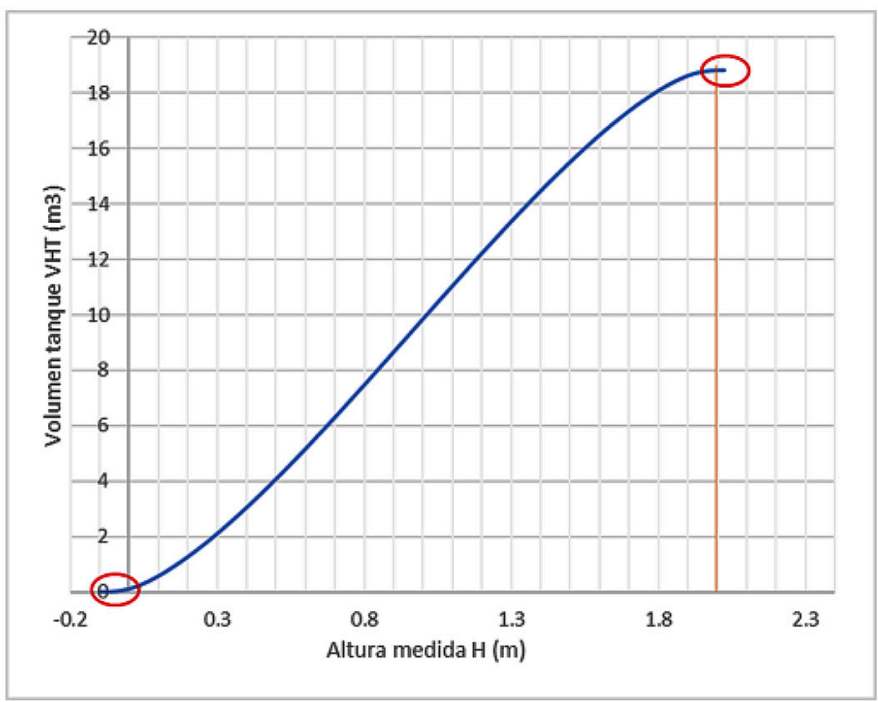

Figura 6. Volumen del tanque en función de la altura.

Fuente: elaboración propia.

Cabe comentar que se probaron las ecuaciones (18) y (19) para calcular el volumen en el Caso III, confirmando que se obtienen resultados idénticos al método que usa las ecuaciones (13) y (17). El resultado se puede observar en la Tabla 2.

Tabla 2. Aplicación de las Ec. (18) y (19) al caso $\mathrm{E} / \mathrm{L}=0,02$ y u/L = 0,2. Fuente: elaboración propia

\begin{tabular}{|c|c|c|c|c|c|c|c|c|c|c|c|}
\hline $\mathrm{H}$ & $\mathrm{H}(0,5)$ & h1 & $\mathrm{h} 2$ & h1' & Q(h1') & h2' & $\mathrm{Q}\left(\mathrm{h} 2{ }^{\prime}\right)$ & Q(h2')-Q(h1') & $\begin{array}{c}\text { Corr } \\
\text { Caso III }\end{array}$ & VHT (m3) & Comentario \\
\hline 1,904 & 1,940 & 1,880 & 2,000 & 0,000 & 0,000 & 0,120 & 0,00371 & 0,003714 & 0,000 & 18,6639 & \\
\hline 1,964 & 2,000 & 1,940 & 2,060 & 0,000 & 0,000 & 0,060 & 0,00066 & 0,000661 & 0,000 & 18,8165 & \\
\hline 2,000 & 2,036 & 1,976 & 2,096 & 0,000 & 0,000 & 0,024 & $6,713 \mathrm{E}-05$ & $6,713 \mathrm{E}-05$ & 0,000 & 18,8462 & \\
\hline 2,024 & 2,060 & 2,000 & 2,120 & 0,000 & 0,000 & 0,000 & 0,00000 & 0,00000 & 0,000 & 18,8496 & tq lleno \\
\hline
\end{tabular}

Los cambios con respecto al Tabla 1 son: los valores diferentes para $\mathrm{h}_{1}{ }^{\prime} \mathrm{y} \mathrm{h}_{2}{ }^{\prime}$, este último calculado con la ecuación (18); que no hay aplicación de la ecuación (17) para corregir el volumen, y que el volumen $\mathrm{V}_{\mathrm{HT}}$ es calculado a partir de la ecuación (19). Se puede comprobar que los resultados finales son idénticos a los del Tabla 1.

Una observación interesante es la variación en el volumen cuando la altura cambia en un centímetro. El cambio de volumen es mínimo en los extremos y máximo para el tanque medio lleno. Usando los datos anteriores podemos generar el gráfico correspondiente, que se ilustra en la Figura 7. Se aprecia un máximo de $0,12 \mathrm{~m}^{3}$ o 120 litros (por centímetro). Considerando que la capacidad 
del tanque del ejemplo es de 18846 litros, esto representa un 0,64\% de la capacidad total. Esa variación es muy elevada, lo cual justificaría el por qué se suelen generar las tablas para este tipo de tanques en milímetros y no en centímetros, como una forma de reducir la incertidumbre.

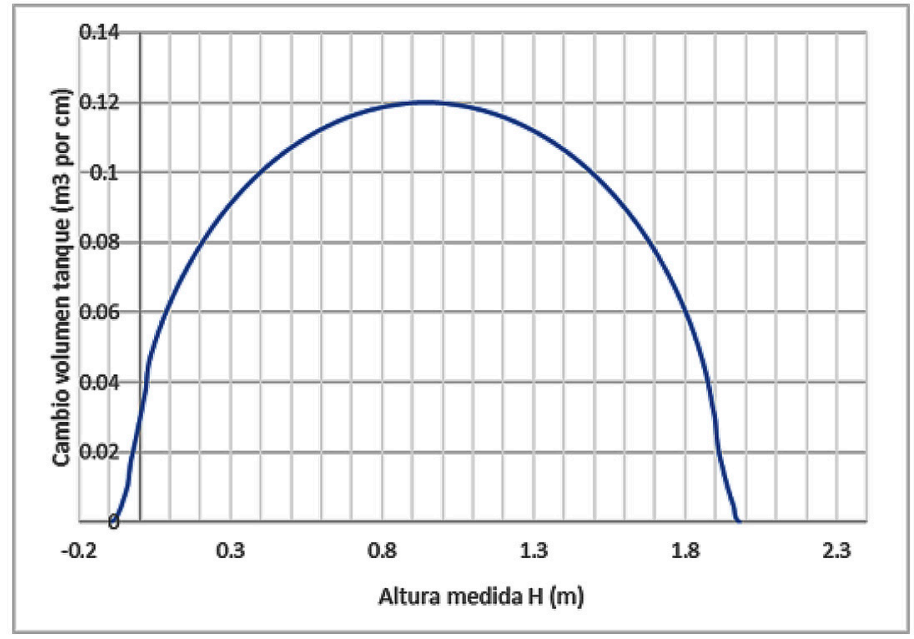

Figura 7. Cambio de volumen por un cambio de un centímetro en la altura.Fuente: elaboración propia.

Como segundo ejemplo, se calculará la curva de porcentaje de corrección en función de h/D para $\mathrm{E} / \mathrm{D}=0,06 \mathrm{y} \mathrm{u} / \mathrm{L}=0,5$, la cual se comparará con la curva correspondiente de la Figura A-1 de la norma ISO 12917:2002. Para ello se utiliza el método ilustrado en el primer ejemplo, por lo que solo se presentan los valores de $\mathrm{V}_{\mathrm{HT}}$ en el Tabla 3 , así como los valores de $\mathrm{V}_{\mathrm{H}}$ y del porcentaje de corrección, calculados mediante las ecuaciones (1), (9) y (10).

Tabla 3. Corrección al volumen para E/D = 0,06. Fuente: elaboración propia.

\begin{tabular}{cccc}
\hline $\mathrm{h} / \mathrm{D}$ & $\mathrm{V}_{\mathrm{HT}}\left(\mathrm{m}^{3}\right)$ & $\mathrm{V}_{\mathrm{H}}\left(\mathrm{m}^{3}\right)$ & $\%$ corr \\
\hline 0,000 & 0,0330407 & 0 & $0,17529 \%$ \\
0,001 & 0,0358555 & 0,0010116 & $0,18485 \%$ \\
0,005 & 0,0485225 & 0,0112967 & $0,19749 \%$ \\
0,010 & 0,0676782 & 0,0319038 & $0,18979 \%$ \\
0,050 & 0,3674629 & 0,3523554 & $0,08015 \%$ \\
0,100 & 0,9906474 & 0,9810067 & $0,05115 \%$ \\
0,200 & 2,6891785 & 2,6837713 & $0,02869 \%$ \\
0,500 & 9,4247780 & 9,4247780 & $0,00000 \%$ \\
0,800 & 16,160377 & 16,165785 & $-0,02869 \%$ \\
0,900 & 17,858909 & 17,868549 & $-0,05115 \%$ \\
0,950 & 18,482093 & 18,497200 & $-0,08015 \%$ \\
0,990 & 18,781878 & 18,817652 & $-0,18979 \%$ \\
0,995 & 18,801033 & 18,838259 & $-0,19749 \%$ \\
0,999 & 18,813700 & 18,848544 & $-0,18485 \%$ \\
1,000 & 18,816515 & 18,849556 & $-0,17529 \%$ \\
\hline
\end{tabular}


Del tabla anterior se observa que la corrección es simétrica con respecto al valor $\mathrm{h} / \mathrm{D}=0,5 \mathrm{y}$ que es aditiva para $\mathrm{h} / \mathrm{D}<0,5$ y sustractiva para $\mathrm{h} / \mathrm{D}>0,5$. Esto es conforme con el análisis realizado al presentar la Figura 3 y permite reducir a la mitad los cálculos y el tamaño de la tabla. Es importante notar que el porcentaje de corrección es con respecto a $\mathrm{V}_{\mathrm{F}}$, el volumen total del tanque. Esto determina que la corrección sea muy importante en los extremos del tanque, del orden de 30 o 40 litros; asimismo, no se debe confundir este cálculo con una estimación típica de error (que usaría $\mathrm{V}_{\mathrm{H}}$ en vez de $\mathrm{V}_{\mathrm{F}}$ ). El cálculo se hace así porque es la forma en que está definido en la norma ISO 12917:2002.

Si se añaden puntos adicionales suficientes para permitir la interpolación lineal en escala logarítmica, se obtienen el Tabla 4 y la Figura 8, los cuales son coincidentes en una forma total con la curva correspondiente de la norma ISO 12917:2002, con la ventaja que en el presente caso es posible obtener valores con mayor número de decimales y por tanto más precisos. Como se comentó anteriormente, las curvas de la Figura 1 se obtuvieron por el método aquí descrito, por comparación es posible confirmar que dicha figura es idéntica a la A-1 de la norma ISO 12917:2002.

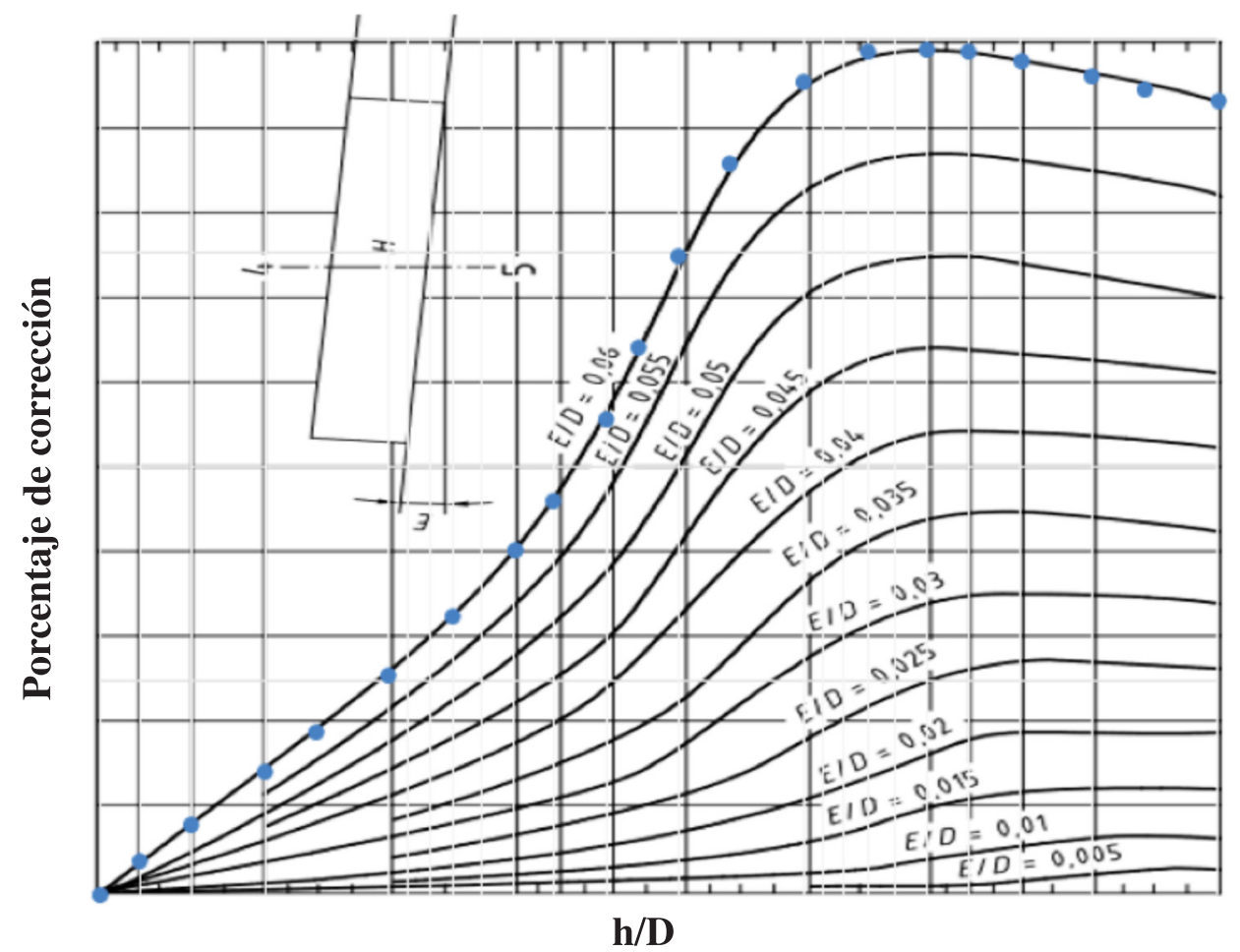

Figura 8. Porcentaje de corrección a $\mathrm{V}_{\mathrm{H}}$ para $\mathrm{E} / \mathrm{D}=0,06$. Comparación entre el método propuesto (puntos azules) y la norma (líneas continuas). Fuente: elaboración propia a partir de la Figura A-1 de la norma ISO 12917-2002.

La Figura 8 anterior, también demuestra que el método propuesto es equivalente al método establecido en la versión más reciente de la norma ISO 12917 (versión 2017), pues dicho método debe dar los mismos valores que la Figura A-1 a la cual reemplaza.

El método propuesto, que es matemáticamente exacto, permite el cálculo de la corrección del volumen con cualquier cantidad de decimales. El número de decimales está asociado a la 
estimación de las correcciones para tanques muy grandes e inclinaciones muy pequeñas. Por ejemplo, se puede observar en el Anexo A.1 que, para $\mathrm{E} / \mathrm{D}=0,005 \mathrm{y} \mathrm{h} / \mathrm{D}=0,4$, el valor de la corrección es de $0,0000541 \%$. Por una razón similar, Coats (1948) presenta sus resultados con nueve cifras significativas, mientras que la norma vigente en la Subsección 10.1 indica que " $A$ menos que se especifique de otra manera, el volumen se expresará con una precisión de cinco dígitos significativos" (ISO, 2017). En los Anexos A.1 y A.2 se presentan, con siete decimales, dos tablas del porcentaje de corrección al volumen del tanque horizontal para diferentes valores de altura h/D y diferentes valores de inclinación E/D, equivalentes a la Figura A-1 de la norma ISO 12917:2002. La primera de las tablas presenta los valores de h/D según una escala logarítmica, con la cual se debe usar interpolación lineal usando logaritmos de h/D; para permitir el uso de interpolación lineal sin cambio de variables, la segunda tabla presenta los valores de h/D según una escala lineal. Como en este caso se requiere incluir aproximadamente el doble de valores de h/D, la tabla del Anexo A.2 está dividida en dos partes, los Anexos A.2.A y A.2.B. En el caso que se requieran valores interpolados con alta exactitud, entonces la interpolación lineal no se recomienda: en su lugar, se recomienda usar otros métodos como diferencias divididas (Coats, 1948), interpolación cuadrática o spline cúbico.

Tabla 4. Porcentajes de corrección a VH para E/D = 0,06.

Fuente: elaboración propia.

\begin{tabular}{cccc}
\hline $\mathrm{h} / \mathrm{D}$ & $\%$ corr & $\mathrm{h} / \mathrm{D}$ & $\%$ corr \\
\hline 0,000 & $0,17529 \%$ & 0,025 & $0,12737 \%$ \\
0,001 & $0,18485 \%$ & 0,030 & $0,11093 \%$ \\
0,0015 & $0,18810 \%$ & 0,040 & $0,09202 \%$ \\
0,002 & $0,19071 \%$ & 0,050 & $0,08015 \%$ \\
0,003 & $0,19443 \%$ & 0,070 & $0,06491 \%$ \\
0,004 & $0,19658 \%$ & 0,100 & $0,05115 \%$ \\
0,005 & $0,19749 \%$ & 0,150 & $0,03752 \%$ \\
0,007 & $0,19644 \%$ & 0,200 & $0,02869 \%$ \\
0,010 & $0,18979 \%$ & 0,300 & $0,01668 \%$ \\
0,015 & $0,17098 \%$ & 0,400 & $0,00780 \%$ \\
0,020 & $0,14857 \%$ & 0,500 & $0,00000 \%$ \\
\hline
\end{tabular}

Este estudio finaliza con un comentario con respecto a la notación empleada en la norma vigente. La norma ISO 12917:2002 basa la corrección por inclinación en las variables H, D, E las cuales han sido utilizadas en este estudio, junto con otras variables cuyo significado es claro (L, h, etc.). En la revisión de la norma del año 2017, se hizo un extenso cambio de variables que no contribuye a hacer el procedimiento de corrección más claro: por ejemplo, L se cambió por W, H' se cambió por 1 pero H se cambió por h, entre otros. La siguiente tabla resume la relación entre las variables del método propuesto en este estudio y las variables equivalentes de la norma vigente. 
La norma vigente también incluye una variable $\delta H_{g}$ en las ecuaciones, que representa el espesor de la placa (dip plate) donde se asienta la plomada cuando se realiza la medición de la profundidad de líquido en el tanque. Este espesor se puede incluir o sumar a la medición de la profundidad -como se indica en la Figura 6 de la norma-, o bien se puede considerar por separado, tal como hace la norma a nivel de ecuaciones. Este es otro ejemplo puntual de falta de claridad de la norma.

Tabla 5. Relación entre las variables de este estudio y las de la norma ISO 12917:2017. Fuente: elaboración propia.

\begin{tabular}{cc}
\hline Este estudio & Norma ISO 12917:2017 \\
\hline$\gamma$ & $\gamma$ \\
$L$ & $W_{c}=W$ \\
$u$ & $W_{c}-W_{g}$ \\
$L-u$ & $W_{g}$ \\
$H$ & $h$ \\
$H^{\prime}$ & $L$ \\
$h_{1}$ & $h_{t}$ \\
$h_{2}$ & $h_{b}$ \\
$E$ & $W \cdot \tan \gamma$ \\
$(L-u) \cdot \tan \gamma$ & $h_{b e}$ \\
\hline
\end{tabular}

\section{CONCLUSIONES}

Este estudio logra presentar de forma clara un método analítico, matemáticamente exacto y explícito para implementar la corrección por inclinación que se describe en la ISO 12917:2017. El método es equivalente al que propone la norma y corrige algunos errores de dicho documento.

Se presentan tablas numéricas con valores de corrección por inclinación, compatibles con la figura A-1 de la norma ISO 12917:2002 y con el método propuesto en la norma vigente, con lo cual se evita hacer interpolaciones en un gráfico. Estas tablas son novedosas y no se han reportado antes en la literatura.

Se presentan las ecuaciones correspondientes que permiten la implementación de la corrección por inclinación en programas de computadora para el cálculo de las tablas de calibración. Las ecuaciones son conformes con la nomenclatura de la norma ISO 12917:2002 y son compatibles con los métodos de cálculo sugeridos por la norma vigente, sin los errores dimensionales o de otro tipo que se han detectado en la norma.

Se interpreta y aclara por qué la norma ISO 12917:2002 solo presenta un gráfico con valores de corrección y asume que el punto de medición está ubicado en el punto medio del tanque. Se explica cómo actuar para aquellos casos en que el punto de medición no está en dicho punto medio. Asimismo, se aclaran los métodos que se deben aplicar en los casos en que la fórmula explicita del caso típico se indefine. 


\section{NOMENCLATURA}

Mayúsculas
A Área del tanque, $\mathrm{m}^{2}$
D Diámetro del tanque, $m$
E Elevación vertical debida a la inclinación, m
$\mathrm{H}$ Altura medida en punto de medición, $\mathrm{m}$
H' Altura real medida en punto de medición, m
L Longitud horizontal o largo del tanque, $\mathrm{m}$
L' Proyección horizontal de la longitud, m
Q Función auxiliar, adim
$\mathrm{R}$ Radio del tanque, $\mathrm{m}$
V Volumen de líquido o del tanque, $\mathrm{m}^{3}$

\section{Minúsculas}

h Altura de líquido, $m$

h' Altura complementaria, m

1 Posición horizontal en el tanque, $\mathrm{m}$

u Posición del punto de medición, m

\section{Subíndices}

1 Posición 1, nivel de líquido más bajo

2 Posición 2, nivel de líquido más alto

0,5 Posición media del tanque

F Tanque lleno (full)

$\mathrm{H}$ Tanque horizontal, no inclinado

HTTanque horizontal inclinado (tilted)

1 Posición horizontal en tanque

Letras griegas

$\Delta$ Cambio, final menos inicial

$\pi$ Constante $=3,14159265 \ldots$ 


\section{REFERENCIAS}

Alonso-Preciado, L., Suárez-Piña, W., Zaldívar-Chacón, J. (2008). Corrección de los errores por inclinación en la calibración de tanques cilíndricos nominalmente horizontales. Boletín Científico Técnico INIMET, (2), 7-14. Disponible en https://www.redalyc.org/articulo.oa?id=223015192003

American Petroleum Institute. (API). (1965). Standard Method for Measurement and Calibration of Horizontal Tanks. Washington D. C.: American Petroleum Institute.

American Petroleum Institute. (2014). (API). Manual of Petroleum Measurement Standards, Chapter 2.2E: Petroleum and Liquid Petroleum Products - Calibration of Horizontal Cylindrical Tanks - Part 1: Manual Methods, First Edition, Includes Errata (2009). Washington D. C.: American Petroleum Institute.

Coats, W. L. (1948). Calibration of Cylindrical Tanks with Axis Inclined. Journal of the Institute of Petroleum, 34(297), 627-646.

International Standardization Organization. (2002). ISO 12917 Petroleum and liquid petroleum products -Calibration of horizontal cylindrical tanks -- Part 1: Manual methods. Ginebra: ISO.

International Standardization Organization. (2017). ISO 12917 Petroleum and liquid petroleum products -Calibration of horizontal cylindrical tanks -- Part 1: Manual methods. Ginebra: ISO.

Khaisongkram, W. y Banjerdpongchai, D. (2004). A combined geometric-volumetric calibration of inclined cylindrical underground storage tanks using the regularized least-squares method. Proceedings of the 2004 IEEE International Conference on Control Applications, 1515-1520. Taipei, Taiwan: IEEE. DOI: 10.1109/CCA.2004.1387590

Li, C.; Yuan, Y.; Song, L.; Tan, Y.; Wang, G. (2013). Mathematical Model Based on BP Neural Network Algorithm for the Deflection Identification of Storage Tank and Calibration of Tank Capacity Chart. Abstract and Applied Analysis, 1-13. DOI: 10.1155/2013/923036.

Xie, W., Wang, X., Cui, H. y Chen, J. (2012). Optimization Model of Oil-Volume Marking with Tilted Oil Tank. Open Journal of Optimization, 1, 20-24. DOI: 10.4236/ojop.2012.12004 


\section{ANEXOS}

\section{Anexo A.1 - Corrección al volumen de un tanque horizontal por inclinación $E / D$}

\begin{tabular}{|c|c|c|c|c|c|c|c|c|c|c|c|c|c|c|}
\hline & & E/D & 0.060 & 0.055 & 0.050 & 0.045 & 0.040 & 0.035 & 0.030 & 0.025 & 0.020 & 0.015 & 0.010 & 0.005 \\
\hline \multicolumn{15}{|c|}{$h / D$} \\
\hline 0.999 & 001 & & $0.1848525 \%$ & $0.1628956 \%$ & $0.1418430 \%$ & $0.1217392 \%$ & $1026354 \%$ & $0845913 \%$ & $.0676785 \%$ & $0.0519844 \%$ & $.0376199 \%$ & $0.0247336 \%$ & $0.0135447 \%$ & .0044684 \\
\hline 0.998 & 0.002 & & $1907127 \%$ & $0.1681980 \%$ & $0.1465653 \%$ & $0.1258568 \%$ & $0.1061210 \%$ & $.0874146 \%$ & $.0698057 \%$ & $0.0533791 \%$ & $0.0382458 \%$ & $0.0245660 \%$ & $0.0126221 \%$ & $0.0032559 \%$ \\
\hline 0.997 & .003 & & $32 \%$ & $1713970 \%$ & $1492264 \%$ & $1279626 \%$ & $1076537 \%$ & $0883571 \%$ & $0701434 \%$ & $0.0531047 \%$ & $.0373713 \%$ & $0.0231581 \%$ & $.0109349 \%$ & .0024861 \\
\hline 0.996 & 0.004 & & $1 \%$ & $730614 \%$ & 15039 & $1286294 \%$ & $.1078098 \%$ & $.0879997 \%$ & $0692787 \%$ & $0.0517575 \%$ & $0.0356079 \%$ & $0.0211495 \%$ & $0.0091845 \%$ & 0.0021181 \\
\hline 0.995 & 0.005 & & $9 \%$ & $5253 \%$ & $0.1504126 \%$ & $1961 \%$ & $069312 \%$ & $884 \%$ & $.0675637 \%$ & $.0496987 \%$ & $0.0333313 \%$ & $0189415 \%$ & $.0078294 \%$ & $.0018802 \%$ \\
\hline 0.994 & 0.006 & & $0.1973851 \%$ & $0.1730185 \%$ & $0.1495056 \%$ & $0.1268965 \%$ & $0.1052547 \%$ & $0.0846645 \%$ & $0.0652453 \%$ & $0.0471838 \%$ & $0.0308104 \%$ & $0.0168278 \%$ & $0.0070011 \%$ & $0.0017084 \%$ \\
\hline 0.993 & 0.007 & & $0.1964401 \%$ & $0.1717131 \%$ & $0.1478494 \%$ & $0.1249067 \%$ & $0.1029596 \%$ & $0.0821108 \%$ & $0.0625122 \%$ & $0.0444096 \%$ & $0.0282553 \%$ & $0.0150411 \%$ & $0.0064117 \%$ & $0.0015763 \%$ \\
\hline 0.992 & 0.008 & & $0.1947884 \%$ & $0.1697448 \%$ & $0.1455819 \%$ & $0.1223666 \%$ & $0.1001885 \%$ & $0.0791742 \%$ & $0.0595163 \%$ & $0.0415360 \%$ & $0.0258382 \%$ & $0.0137506 \%$ & $0.0059554 \%$ & $0.0014706 \%$ \\
\hline 0.991 & 0.009 & & $1925404 \%$ & $1672255 \%$ & $0.1428163 \%$ & $0.1193918 \%$ & $0.0970598 \%$ & $0.0759766 \%$ & $0.0563850 \%$ & $0.0386979 \%$ & $0.0237060 \%$ & $0.0128054 \%$ & $0.0055861 \%$ & 0.0 \\
\hline 0.99 & 01 & & $1 \%$ & $\%$ & $8 \%$ & $\%$ & $744 \%$ & $0726226 \%$ & $8 \%$ & $0121 \%$ & $8870 \%$ & $2 \%$ & $1 \%$ & $0.0013095 \%$ \\
\hline & & & & & & & & & & & & & & \\
\hline 0.97 & 0.03 & & $0.1109272 \%$ & $0.0921605 \%$ & $0.0755003 \%$ & $0.0607196 \%$ & $0.0476928 \%$ & $.0363355 \%$ & $.0265871 \%$ & $0.0184022 \%$ & $0.0117464 \%$ & $0.0065941 \%$ & $0.0029266 \%$ & $0.0007310^{\circ}$ \\
\hline 0.96 & 0.04 & & $0.0920178 \%$ & $0.0769632 \%$ & $0.0633499 \%$ & $0.0511337 \%$ & $0.0402795 \%$ & $0.0307587 \%$ & $0.0225485 \%$ & $0.0156300 \%$ & $0.0099885 \%$ & $0.0056121 \%$ & $0.0024923 \%$ & 0.0006228 \\
\hline 0.95 & 0.05 & & $0.0801473 \%$ & $0.0671615 \%$ & $0.0553700 \%$ & $0.0447529 \%$ & $0.0352933 \%$ & $0.0269770 \%$ & $0.0197920 \%$ & $0.0137283 \%$ & $0.0087777 \%$ & $0.0049338 \%$ & $0.0021917 \%$ & $0.0005477^{\circ}$ \\
\hline 0.94 & 0.06 & & $0.0715624 \%$ & $0.0600207 \%$ & $0.0495212 \%$ & $0.0400525 \%$ & $0.0316048 \%$ & $0.0241697 \%$ & $0.0177399 \%$ & $0.0123092 \%$ & $0.0078726 \%$ & $0.0044260 \%$ & $0.0019664 \%$ & $0.0004915 \%$ \\
\hline 0.93 & 0.07 & & $0.0649071 \%$ & $0.0544660 \%$ & $9580 \%$ & $0.0363760 \%$ & $0.0287136 \%$ & $0219651 \%$ & $.0161258 \%$ & $1916 \%$ & $90 \%$ & $.0040254 \%$ & $886 \%$ & $1 \%$ \\
\hline 0.92 & 0.08 & & $0.0595142 \%$ & $0.0499562 \%$ & $0.0412471 \%$ & $0.0333817 \%$ & $0.0263558 \%$ & $.0201653 \%$ & $0.0148068 \%$ & $.0102776 \%$ & $0.0065751 \%$ & $0.0036974 \%$ & $0.0016429 \%$ & $0.0004107^{\circ}$ \\
\hline 0.91 & 0.09 & & $0.0550053 \%$ & $0.0461811 \%$ & 0.03 & $0.0308702 \%$ & $0.0243765 \%$ & $.0186533 \%$ & $.0136982 \%$ & $0.0095089 \%$ & $0.0060838 \%$ & $0.0034213 \%$ & $.0015203 \%$ & $0.00038 \mathrm{C}$ \\
\hline 0.9 & 0.1 & & $0.0511458 \%$ & $0.0429472 \%$ & $0.0354714 \%$ & $0.0287157 \%$ & $0.0226776 \%$ & $173549 \%$ & $27457 \%$ & $0.0088484 \%$ & $5 \%$ & $0031839 \%$ & $\%$ & 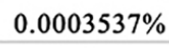 \\
\hline 0.8 & 0.2 & & $0.0286858 \%$ & $0.0240990 \%$ & $0.0199126 \%$ & 0.01 & 0.01 & $26 \%$ & $13 \%$ & $7 \%$ & $0.0031836 \%$ & $.0017906 \%$ & $.0007958 \%$ & $989 \%$ \\
\hline 0.7 & 0.3 & & $\%$ & $\%$ & 0. & 0. & $\%$ & $\%$ & $\%$ & $\%$ & $1 \%$ & $\%$ & $\%$ & $58 \%$ \\
\hline \multirow[t]{2}{*}{0.6} & 0.4 & & $0.0078015 \%$ & $0.0065548 \%$ & $0.0054168 \%$ & $0.0043872 \%$ & $0.0034662 \%$ & $0.0026537 \%$ & $.0019495 \%$ & $0.0013538 \%$ & $0.0008664 \%$ & $0.0004873 \%$ & $0.0002166 \%$ & $0.0000541 \%$ \\
\hline & 0.5 & & $0.0000000 \%$ & $0.0000000 \%$ & $0.0000000 \%$ & $0.0000000 \%$ & $0.0000000 \%$ & $0.0000000 \%$ & $0.0000000 \%$ & $0.0000000 \%$ & $0.0000000 \%$ & $0.0000000 \%$ & $0.0000000 \%$ & $0.0000000 \%$ \\
\hline
\end{tabular}

La corrección es aditiva para h/D de 0 a 0,5 y sustractiva de 0,5 a 1 . 


\section{Anexo A.2.A - Corrección al volumen de un tanque horizontal por inclinación $E / D$}

\begin{tabular}{|c|c|c|c|c|c|c|c|c|c|c|c|c|c|c|}
\hline & & E/D & 0.060 & 0.055 & 0.050 & 0.045 & 0.040 & 0.035 & 0.030 & 0.025 & 0.020 & 0.015 & 0.010 & 0.005 \\
\hline \multicolumn{2}{|c|}{ h/D } & & & & & & & & & & & & & \\
\hline 0.99 & 0.01 & & $0.1897891 \%$ & $0.1642495 \%$ & $0.1396488 \%$ & $0.1160804 \%$ & $0.0936744 \%$ & $0.0726226 \%$ & $0.0532278 \%$ & $0.0360121 \%$ & $0.0219870 \%$ & $0.0120472 \%$ & $0.0052781 \%$ & 0.0013095 \\
\hline 0.98 & 0.02 & & $0.1485740 \%$ & $0.1236295 \%$ & $0.1004663 \%$ & $0.0794871 \%$ & $0.0612889 \%$ & $0.0461953 \%$ & $0.0335653 \%$ & $0.0231159 \%$ & $0.0147014 \%$ & $0.0082314 \%$ & $0.0036468 \%$ & $0.0009100 \%$ \\
\hline 0.97 & 0.03 & & $0.1109272 \%$ & $0.0921605 \%$ & $0.0755003 \%$ & $0.0607196 \%$ & $0.0476928 \%$ & $0.0363355 \%$ & $0.0265871 \%$ & $0.0184022 \%$ & $0.0117464 \%$ & $0.0065941 \%$ & $0.0029266 \%$ & $0.0007310 \%$ \\
\hline 0.96 & 0.04 & & $0.0920178 \%$ & $0.0769632 \%$ & $0.0633499 \%$ & $0.0511337 \%$ & $0.0402795 \%$ & $.0307587 \%$ & $0.0225485 \%$ & $0.0156300 \%$ & $0.0099885 \%$ & $0.0056121 \%$ & $0.0024923 \%$ & $0.0006228 \%$ \\
\hline 0.95 & 0.05 & & $0.0801473 \%$ & $0.0671615 \%$ & $0553700 \%$ & $0.0447529 \%$ & $0.0352933 \%$ & $9770 \%$ & $197920 \%$ & $0.0137283 \%$ & $0.0087777 \%$ & $\%$ & $0.0021917 \%$ & $0.0005477 \%$ \\
\hline 0.94 & 0.06 & & $0.0715624 \%$ & $0.0600207 \%$ & $0.0495212 \%$ & $0.0400525 \%$ & $0.0316048 \%$ & $0241697 \%$ & $0.0177399 \%$ & $0.0123092 \%$ & $0.0078726 \%$ & 0.00 & $0.0019664 \%$ & 0 . \\
\hline 0.93 & 0.07 & & $0.0649071 \%$ & $0.0544660 \%$ & $.0449580 \%$ & $0.0363760 \%$ & $0.0287136 \%$ & $.0219651 \%$ & $0.0161258 \%$ & $0.0111916 \%$ & $0.0071590 \%$ & $254 \%$ & $7886 \%$ & $4471 \%$ \\
\hline 0.92 & 0.08 & & $0.0595142 \%$ & $0.0499562 \%$ & $0.0412471 \%$ & $0.0333817 \%$ & $0.0263558 \%$ & $0.0201653 \%$ & $0.0148068 \%$ & $0.0102776 \%$ & $0.0065751 \%$ & $0.0036974 \%$ & $0.0016429 \%$ & $0.0004107 \%$ \\
\hline 0.91 & 0.09 & & $0.0550053 \%$ & $0.0461811 \%$ & $0.0381373 \%$ & $0.0308702 \%$ & $0.0243765 \%$ & $0.0186533 \%$ & $0.0136982 \%$ & $0.0095089 \%$ & $0.0060838 \%$ & $0.0034213 \%$ & $0.0015203 \%$ & $0.0003800 \%$ \\
\hline 0.90 & 0.10 & & $0.0511458 \%$ & $0.0429472 \%$ & $0.0354714 \%$ & $0.0287157 \%$ & $0.0226776 \%$ & $0.0173549 \%$ & $0.0127457 \%$ & $0.0088484 \%$ & $0.0056615 \%$ & $.0031839 \%$ & $0.0014149 \%$ & t \\
\hline 0.89 & 0.11 & & $0.0477810 \%$ & $0.0401261 \%$ & $0.0331447 \%$ & $0.0268345 \%$ & $0.0211937 \%$ & $0.0162204 \%$ & $0.0119132 \%$ & $0.0082708 \%$ & $0.0052922 \%$ & $0.0029763 \%$ & $0.0013226 \%$ & $0.0003306 \%$ \\
\hline 0.88 & 0.12 & & $0.0448036 \%$ & $0.0376289 \%$ & $0.0310843 \%$ & $0.0251681 \%$ & $0.0198787 \%$ & $0.0152148 \%$ & $0.0111751 \%$ & $0.0077587 \%$ & $0.0049646 \%$ & $0.0027922 \%$ & $0.0012408 \%$ & $0.0003102 \%$ \\
\hline 0.87 & 0.13 & & $0.0421365 \%$ & $0.0353912 \%$ & $0.0292375 \%$ & $0.0236740 \%$ & $0.0186995 \%$ & $0.0143129 \%$ & $0.0105131 \%$ & $0.0072993 \%$ & $0.0046708 \%$ & $0.0026270 \%$ & $0.0011674 \%$ & $0.0002918 \%$ \\
\hline 0.86 & 0.14 & & $0.0397228 \%$ & $0.0333656 \%$ & $0.0275654 \%$ & $0.0223210 \%$ & $0.0176315 \%$ & $0.0134958 \%$ & $0.0099132 \%$ & $0.0068829 \%$ & $0.0044044 \%$ & $0.0024772 \%$ & $0.0011009 \%$ & $.0002752 \%$ \\
\hline 0.85 & 0.15 & & $0.0375191 \%$ & $0.0315159 \%$ & $0260382 \%$ & $0.0210851 \%$ & $0.0166557 \%$ & $.0127493 \%$ & $0.0093651 \%$ & $0.0065025 \%$ & $0.0041610 \%$ & $0.0023403 \%$ & $0.0010401 \%$ & 0.000260 \\
\hline 0.84 & 0.16 & & $0.0354917 \%$ & $0.0298139 \%$ & $0.0246328 \%$ & $0.0199476 \%$ & $0.0157576 \%$ & $0.0120620 \%$ & $0.0088604 \%$ & $0.0061522 \%$ & $0.0039369 \%$ & $0.0022143 \%$ & $0.0009841 \%$ & $0.0002460 \%$ \\
\hline 0.83 & 0.17 & & $0.0336142 \%$ & $0.0282375 \%$ & $0.0233310 \%$ & $0.0188938 \%$ & $0.0149254 \%$ & $0.0114253 \%$ & $0.0083928 \%$ & $0.0058276 \%$ & $0.0037292 \%$ & $0.0020975 \%$ & $0.0009322 \%$ & $0.0002330 \%$ \\
\hline 0.82 & 0.18 & & $0.0318650 \%$ & $0.0267688 \%$ & $0.0221179 \%$ & $0.0179118 \%$ & $0.0141500 \%$ & $0.0108318 \%$ & $0.0079569 \%$ & $0.0055250 \%$ & $0.0035357 \%$ & $0.0019887 \%$ & $0.0008838 \%$ & $0.0002209 \%$ \\
\hline 0.81 & 0.19 & & $0.0302270 \%$ & $0.0253933 \%$ & $0.0209818 \%$ & $0.0169920 \%$ & $0.0134235 \%$ & $0.0102759 \%$ & $0.0075486 \%$ & $0.0052415 \%$ & $0.0033543 \%$ & $0.0018867 \%$ & $0.0008385 \%$ & .000209 \\
\hline 0.80 & 0.20 & & $36858 \%$ & $0.0240990 \%$ & $126 \%$ & $0.0161264 \%$ & $0.0127399 \%$ & $0.0097526 \%$ & $0.0071643 \%$ & $0.0049747 \%$ & $0.0031836 \%$ & $906 \%$ & $0007958 \%$ & $989 \%$ \\
\hline 0.79 & 0.21 & & $0.0272296 \%$ & $0.0228759 \%$ & $0.0189023 \%$ & $0.0153084 \%$ & $0.0120937 \%$ & $.0092581 \%$ & $0.0068011 \%$ & $0.0047225 \%$ & $0.0030222 \%$ & $.0016999 \%$ & $0.0007555 \%$ & $0.0001889 \%$ \\
\hline 0.78 & 0.22 & & $0.0258482 \%$ & $0.0217157 \%$ & $0.0179438 \%$ & $0.0145323 \%$ & $0.0114807 \%$ & $0.0087889 \%$ & $0.0064565 \%$ & $0.0044833 \%$ & $0.0028691 \%$ & $0.0016138 \%$ & $0.0007172 \%$ & $0.0001793 \%$ \\
\hline 0.77 & 0.23 & & $0.0245332 \%$ & $0.0206112 \%$ & $0.0170313 \%$ & $0.0137934 \%$ & $0.0108971 \%$ & $0.0083422 \%$ & $0.0061283 \%$ & $0.0042554 \%$ & $0.0027233 \%$ & $0.0015318 \%$ & $0.0006808 \%$ & $0.0001702 \%$ \\
\hline 0.76 & 0.24 & & $0.0232774 \%$ & $0.0195563 \%$ & $0.0161598 \%$ & $0.0130877 \%$ & $0.0103396 \%$ & $0.0079155 \%$ & $0.0058149 \%$ & $0.0040378 \%$ & $0.0025840 \%$ & $0.0014534 \%$ & $0.0006460 \%$ & $0.0001615^{\circ}$ \\
\hline 0.75 & 0.25 & & $0.0220744 \%$ & $0.0185458 \%$ & $0.0153249 \%$ & $0.0124116 \%$ & $0.0098056 \%$ & $0.0075067 \%$ & $0.0055146 \%$ & $0.0038293 \%$ & $0.0024506 \%$ & $0.0013784 \%$ & $0.0006126 \%$ & $0.0001531 \%$ \\
\hline
\end{tabular}

La corrección es aditiva para h/D de 0 a 0,5 y sustractiva de 0,5 a 1 . 
Anexo A.2.B. - Corrección al volumen de un tanque horizontal por inclinación $E / D$

\begin{tabular}{|c|c|c|c|c|c|c|c|c|c|c|c|c|c|c|}
\hline & & E/D & 0.060 & 0.055 & 0.050 & 0.045 & 0.040 & 0.035 & 0.030 & 0.025 & 0.020 & 0.015 & 0.010 & 0.005 \\
\hline \multicolumn{15}{|c|}{ h/D } \\
\hline 0.74 & 0.26 & & $0.0209188 \%$ & $0.0175750 \%$ & $0.0145229 \%$ & $0.0117621 \%$ & $0.0092925 \%$ & $0.0071139 \%$ & $0.0052261 \%$ & $0.0036290 \%$ & $0.0023224 \%$ & $0.0013063 \%$ & $0.0005806 \%$ & $0.0001451 \%$ \\
\hline 0.73 & 0.27 & & $0.0198059 \%$ & $0.0166401 \%$ & $0.0137504 \%$ & $0.0111366 \%$ & $0.0087983 \%$ & $0.0067356 \%$ & $0.0049482 \%$ & $0.0034360 \%$ & $0.0021989 \%$ & $0.0012369 \%$ & $0.0005497 \%$ & $0.0001374 \%$ \\
\hline 0.72 & 0.28 & & $0.0187314 \%$ & $0.0157375 \%$ & $0.0130046 \%$ & $0.0105326 \%$ & $0.0083212 \%$ & $0.0063704 \%$ & $0.0046799 \%$ & $0.0032497 \%$ & $0.0020797 \%$ & $0.0011698 \%$ & $0.0005199 \%$ & $0.0001300 \%$ \\
\hline 0.71 & 0.29 & & $0.0176917 \%$ & $0.0148640 \%$ & $0.0122829 \%$ & $0.0099481 \%$ & $0.0078595 \%$ & $0.0060169 \%$ & $0.0044203 \%$ & $0.0030694 \%$ & $0.0019643 \%$ & $0.0011049 \%$ & $0.0004911 \%$ & $0.0001228 \%$ \\
\hline 0.70 & 0.30 & & $0.0166834 \%$ & $0.0140170 \%$ & $0.0115830 \%$ & $0.0093813 \%$ & $0.0074117 \%$ & $0.0056741 \%$ & $0.0041685 \%$ & $0.0028946 \%$ & $0.0018524 \%$ & $0.0010420 \%$ & $0.0004631 \%$ & $0.0001158 \%$ \\
\hline 0.69 & 0.31 & & $0.0157036 \%$ & $0.0131939 \%$ & $0.0109028 \%$ & $0.0088304 \%$ & $0.0069765 \%$ & $0.0053410 \%$ & $0.0039237 \%$ & $0.0027247 \%$ & $0.0017437 \%$ & $0.0009808 \%$ & $0.0004359 \%$ & $0.0001090 \%$ \\
\hline 0.68 & 0.32 & & $0.0147497 \%$ & $0.0123925 \%$ & $0.0102406 \%$ & $0.0082941 \%$ & $0.0065528 \%$ & $0.0050166 \%$ & $0.0036855 \%$ & $0.0025592 \%$ & $0.0016378 \%$ & $0.0009212 \%$ & $0.0004094 \%$ & $0.0001024 \%$ \\
\hline 0.67 & 0.33 & & $0.0138193 \%$ & $0.0116108 \%$ & $0.0095947 \%$ & $0.0077710 \%$ & $0.0061396 \%$ & $0.0047003 \%$ & $0.0034530 \%$ & $0.0023978 \%$ & $0.0015345 \%$ & $0.0008631 \%$ & $0.0003836 \%$ & $0.0000959 \%$ \\
\hline 0.66 & 0.34 & & $0.0129102 \%$ & $0.0108470 \%$ & $0.0089635 \%$ & $0.0072598 \%$ & $0.0057357 \%$ & $0.0043911 \%$ & $0.0032259 \%$ & $0.0022401 \%$ & $0.0014336 \%$ & $0.0008064 \%$ & $0.0003584 \%$ & $0.0000896 \%$ \\
\hline 0.65 & 0.35 & & $0.0120203 \%$ & $0.0100993 \%$ & $0.0083458 \%$ & $0.0067595 \%$ & $0.0053404 \%$ & $0.0040885 \%$ & $0.0030036 \%$ & $0.0020857 \%$ & $0.0013348 \%$ & $0.0007508 \%$ & $0.0003337 \%$ & $0.0000834 \%$ \\
\hline 0.64 & 0.36 & & $0.0111479 \%$ & $0.0093664 \%$ & $0.0077401 \%$ & $0.0062690 \%$ & $0.0049529 \%$ & $0.0037918 \%$ & $0.0027857 \%$ & $0.0019344 \%$ & $0.0012380 \%$ & $0.0006963 \%$ & $0.0003095 \%$ & $0.0000774 \%$ \\
\hline 0.63 & 0.37 & & $0.0102914 \%$ & $0.0086468 \%$ & $0.0071454 \%$ & $0.0057873 \%$ & $0.0045724 \%$ & $0.0035005 \%$ & $0.0025716 \%$ & $0.0017858 \%$ & $0.0011429 \%$ & $0.0006428 \%$ & $0.0002857 \%$ & $0.0000714 \%$ \\
\hline 0.62 & 0.38 & & $0.0094491 \%$ & $0.0079391 \%$ & $0.0065606 \%$ & $0.0053137 \%$ & $0.0041982 \%$ & $0.0032140 \%$ & $0.0023612 \%$ & $0.0016396 \%$ & $0.0010493 \%$ & $0.0005902 \%$ & $0.0002623 \%$ & $0.0000656 \%$ \\
\hline 0.61 & 0.39 & & $0.0086196 \%$ & $0.0072422 \%$ & $0.0059847 \%$ & $0.0048472 \%$ & $0.0038297 \%$ & $0.0029319 \%$ & $0.0021539 \%$ & $0.0014957 \%$ & $0.0009572 \%$ & $0.0005384 \%$ & $0.0002393 \%$ & $0.0000598 \%$ \\
\hline 0.60 & 0.40 & & $0.0078015 \%$ & $0.0065548 \%$ & $0.0054168 \%$ & $.0043872 \%$ & $0.0034662 \%$ & $0.0026537 \%$ & $0.0019495 \%$ & $0.0013538 \%$ & $0.0008664 \%$ & $0.0004873 \%$ & $0.0002166 \%$ & $0.0000541 \%$ \\
\hline 0.59 & 0.41 & & $0.0069937 \%$ & $0.0058761 \%$ & $0.0048559 \%$ & $.0039330 \%$ & $0.0031073 \%$ & $0.0023789 \%$ & $0.0017477 \%$ & $0.0012136 \%$ & $0.0007767 \%$ & $0.0004369 \%$ & $0.0001942 \%$ & $0.0000485 \%$ \\
\hline 0.58 & 0.42 & & $0.0061948 \%$ & $0.0052049 \%$ & $0.0043012 \%$ & $.0034837 \%$ & $0.0027524 \%$ & $0.0021072 \%$ & $0.0015480 \%$ & $0.0010750 \%$ & $0.0006880 \%$ & $0.0003870 \%$ & $0.0001720 \%$ & $0.0000430 \%$ \\
\hline 0.57 & 0.43 & & $0.0054038 \%$ & $0.0045403 \%$ & $0.0037520 \%$ & $0.0030389 \%$ & $0.0024010 \%$ & $0.0018381 \%$ & $0.0013504 \%$ & $0.0009377 \%$ & $0.0006001 \%$ & $0.0003376 \%$ & $0.0001500 \%$ & $0.0000375 \%$ \\
\hline 0.56 & 0.44 & & $046196 \%$ & $0.0038814 \%$ & $0.0032075 \%$ & $.0025979 \%$ & $0.0020525 \%$ & $0.0015714 \%$ & $0.0011544 \%$ & $0.0008016 \%$ & $0.0005130 \%$ & $0.0002886 \%$ & $0.0001283 \%$ & $0.0000321 \%$ \\
\hline 0.55 & 0.45 & & $0.0038411 \%$ & $0.0032273 \%$ & $0.0026670 \%$ & $.0021601 \%$ & $0.0017066 \%$ & $0.0013066 \%$ & $0.0009599 \%$ & $0.0006666 \%$ & $0.0004266 \%$ & $0.0002399 \%$ & $0.0001066 \%$ & $0.0000267 \%$ \\
\hline 0.54 & 0.46 & & $0.0030673 \%$ & $0.0025771 \%$ & $0.0021297 \%$ & $0.0017249 \%$ & $0.0013628 \%$ & $0.0010433 \%$ & $0.0007665 \%$ & $0.0005323 \%$ & $0.0003406 \%$ & $0.0001916 \%$ & $0.0000852 \%$ & $0.0000213 \%$ \\
\hline 0.53 & 0.47 & & $0.0022972 \%$ & $0.0019301 \%$ & $0.0015950 \%$ & $.0012919 \%$ & $0.0010207 \%$ & $0.0007814 \%$ & $0.0005741 \%$ & $.0003986 \%$ & $0.0002551 \%$ & $.0001435 \%$ & $0.0000638 \%$ & $0.0000159 \%$ \\
\hline 0.52 & 0.48 & & $0.0015299 \%$ & $0.0012855 \%$ & $0.0010623 \%$ & $0.0008604 \%$ & $0.0006798 \%$ & $0.0005204 \%$ & $0.0003823 \%$ & $0.0002655 \%$ & $0.0001699 \%$ & $0.0000956 \%$ & $0.0000425 \%$ & $0.0000106 \%$ \\
\hline 0.51 & 0.49 & & $0.0007645 \%$ & $0.0006423 \%$ & $0.0005308 \%$ & $0.0004299 \%$ & $0.0003397 \%$ & $0.0002601 \%$ & $0.0001910 \%$ & $0.0001327 \%$ & $0.0000849 \%$ & $0.0000478 \%$ & $0.0000212 \%$ & $0.0000053 \%$ \\
\hline 0.50 & 0.50 & & $0.0000000 \%$ & $0.0000000 \%$ & $0.0000000 \%$ & $0.0000000 \%$ & $0.0000000 \%$ & $0.0000000 \%$ & $0.0000000 \%$ & $0.0000000 \%$ & $0.0000000 \%$ & $0.0000000 \%$ & $0.0000000 \%$ & $0.0000000 \%$ \\
\hline
\end{tabular}

La corrección es aditiva para h/D de 0 a 0,5 y sustractiva de 0,5 a 1 . 